\title{
Identification and reproducibility of dietary patterns assessed with a FFQ among women planning pregnancy
}

\section{Lim, SX}

2021-06

Lim , SX, Colega , MT , Ayob , MNM , Robinson, SM , Godfrey , KM , Bernard, JY, Lee , YS , Tan , KH , Yap , F , Shek, LPC , Chong , YS , Eriksson, JG, Chan , JKY, Chan , SY \& Chong, MFF 2021, ' Identification and reproducibility of dietary patterns assessed with a FFQ among women planning pregnancy ' , Public Health Nutrition , vol. 24 , no. 9 , 1368980021001178 , pp. 2437-2446 . https://doi.org/10.1017/S1368980021001178

http://hdl.handle.net/10138/335638

https://doi.org/10.1017/S1368980021001178

cc_by

publishedVersion

Downloaded from Helda, University of Helsinki institutional repository.

This is an electronic reprint of the original article.

This reprint may differ from the original in pagination and typographic detail.

Please cite the original version. 


\title{
Identification and reproducibility of dietary patterns assessed with a FFQ among women planning pregnancy
}

\author{
Shan Xuan Lim', Marjorelee T Colega ${ }^{2}, M$ Na'im M Ayob², Sian M Robinson ${ }^{3,4}$, \\ Keith M Godfrey,6, Jonathan Y Bernard ${ }^{2,7}$ (1), Yung Seng Lee ${ }^{2,8,9}$ (D), Kok Hian Tan ${ }^{10,1} 1$ (1), \\ Fabian Yap 10,12,13, Lynette PC Shek 2,8,9, Yap Seng Chong 2,14, Johan G Eriksson 2,14,15,16, \\ Jerry KY Chan 10,17, Shiao Yng Chan ${ }^{2,14}$ and Mary FF Chong ${ }^{1,2, *}$
}

'Saw Swee Hock School of Public Health, National University of Singapore and National University Health System, Singapore, Singapore: ${ }^{2}$ Singapore Institute for Clinical Sciences, Agency for Science, Technology and Research (A*STAR), Singapore, Singapore: ${ }^{3}$ AGE Research Group, Newcastle University Institute for Translational and Clinical Research, Newcastle upon Tyne, UK: ${ }^{4}$ NIHR Newcastle Biomedical Research Centre, Newcastle University and Newcastle upon Tyne NHS Foundation Trust, Newcastle upon Tyne, UK: ${ }^{5}$ Medical Research Council Lifecourse Epidemiology Unit, University of Southampton, Southampton, UK: ${ }^{6}$ NIHR Southampton Biomedical Research Centre, University Hospital Southampton, NHS Foundation Trust, Southampton, UK: 'Université de Paris, Centre for Research in Epidemiology and StatisticS (CRESS), Inserm, INRAE, Paris, France: ${ }^{8}$ Department of Paediatrics, Yong Loo Lin School of Medicine, National University of Singapore, Singapore, Singapore: ${ }^{9}$ Khoo Teck Puat-National University Children's Medical Institute, National University Hospital, National University Health System, Singapore, Singapore: ${ }^{10}$ Duke-NUS Medical School, Singapore, Singapore: 1'Department of Maternal Fetal Medicine, KK Women's and Children's Hospital, Singapore, Singapore: ${ }^{12}$ Department of Paediatrics, KK Women's and Children's Hospital, Singapore, Singapore: ${ }^{13}$ Lee Kong Chian School of Medicine, Nanyang Technological University, Singapore, Singapore: ${ }^{14}$ Department of Obstetrics and Gynaecology, Yong Loo Lin School of Medicine, National University of Singapore, Singapore, Singapore: ${ }^{15}$ Department of General Practice and Primary Health Care, University of Helsinki and Helsinki University Hospital, Helsinki, Finland: ${ }^{16}$ Folkhälsan Research Center, University of Helsinki, Helsinki, Finland: ${ }^{17}$ Department of Reproductive Medicine, KK Women's and Children's Hospital, Singapore, Singapore

Submitted 26 June 2020: Final revision received 16 February 2021: Accepted 16 March 2021: First published online 22 March 2021

\begin{abstract}
Objective: To identify a posteriori dietary patterns among women planning pregnancy and assess the reproducibility of these patterns in a subsample using two dietary assessment methods.

Design: A semi-quantitative FFQ was administered to women enrolled in the Singapore PREconception Study of long-Term maternal and child Outcomes study. Dietary patterns from the FFQ were identified using exploratory factor analysis (EFA). In a subsample of women ( $n$ 289), 3-d food diaries (3DFD) were also completed and analysed. Reproducibility of the identified patterns was assessed using confirmatory factor analysis (CFA) in the subsample, and goodness of fit of the CFA models was examined using several fit indices. Subsequently, EFA was conducted in the subsample and dietary patterns of the FFQ and the 3DFD were compared. Setting: Singapore.

Participants: 1007 women planning pregnancy (18-45 years)

Results: Three dietary patterns were identified from the FFQ: the 'Fish, Poultry/ Meat and Noodles' pattern was characterised by higher intakes of fish, poultry/ meat and noodles in soup; 'Fast Food and Sweetened Beverages' pattern was characterised by higher intakes of fast food, sweetened beverages and fried snacks; 'Bread, Legumes and Dairy' pattern was characterised by higher intakes of buns/ethnic breads, nuts/legumes and dairy products. The comparative fit indices from the CFA models were 0.79 and 0.34 for the FFQ and 3DFD of the subsample, respectively. In the subsample, three similar patterns were identified in the FFQ while only two for the 3DFD.

Conclusions: Dietary patterns from the FFQ are reproducible within this cohort, providing a basis for future investigations on diet and health outcomes.
\end{abstract}

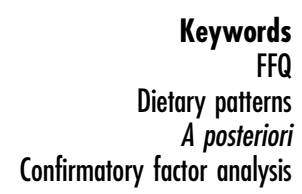
Confirmatory factor analysis

*Corresponding author: Email mary_chong@nus.edu.sg

(c) The Author(s), 2021. Published by Cambridge University Press on behalf of The Nutrition Society 
As milestones of a woman's reproductive lifespan, the stages of preconception, pregnancy and postpartum may bring about distinct dietary changes ${ }^{(1-5)}$. In general, increased intakes of healthier foods (e.g., fruits and vegetables) may be observed when women transit from preconception to pregnancy ${ }^{(2)}$, while a sharp decline in consumption of these foods tended to occur at postpartum, although these remained at higher levels compared with the preconception stage ${ }^{(4,5)}$. A similar trend has been observed locally in Singapore, such that women increased fruit and vegetable intakes but decreased tea/coffee, soft drinks and seafood intakes during pregnancy, while at postpartum they consumed more ginger, garlic, fish and milk-based drinks but less of sweets, chocolate and seafood ${ }^{(1)}$. These dietary changes, seemingly unique to the local Asian population, were found to differ by ethnicity and were based on traditional beliefs, particularly at postpartum ${ }^{(1)}$. However, these data were collected from short questionnaires with pre-defined food items. In order to systematically observe changes in dietary patterns across several time points of a woman's reproductive lifespan and enable diet comparisons to be made, a culturally relevant and validated maternal FFQ is required.

Integrative approaches of examining diets in their entirety have been increasingly used ${ }^{(6,7)}$, such as $a$ posteriori or a priori dietary patterns. Although limited by their population specificity, a posteriori dietary patterns are valuable as they depict actual dietary intakes of populations at different stages across the lifespan ${ }^{(8)}$. Additionally, these unique and well-defined patterns provide realistic representations of consumption patterns in ethnically heterogeneous populations ${ }^{(8)}$. Studies examining a posteriori dietary patterns have observed that the patterns derived were generally qualitatively similar and reasonably valid across different dietary assessment methods ${ }^{(8-15)}$. In patterns identified among women planning pregnancy, a posteriori healthy dietary patterns (e.g., 'Vegetables and Meat'(3), 'Fruit and Low-fat Dairy' $\left.{ }^{\text {(16) }}\right)$ and less healthy dietary patterns (e.g., 'Sweetened Beverages and Sugars' (3), 'High-fat/sugar/takeaway ${ }^{\text {(17) }}$ ) were observed, but the reproducibility of these patterns has not been examined. The few reproducibility studies on dietary patterns of Europeans have shown that the FFQ-derived patterns were reproducible in smaller samples of the same cohort ${ }^{(18,19)}$ and similar dietary patterns were identified using two different dietary assessment methods ${ }^{(15)}$.

To bridge the gap, we aimed to (1) identify a posteriori dietary patterns among an Asian cohort of women planning pregnancy using exploratory factor analysis (EFA) and (2) assess the reproducibility of these patterns in a subsample using two different dietary assessment methods (food frequency questionnaire (FFQ) and 3-d food diary (3DFD)). We hypothesised that the identified patterns would be reproducible in both dietary assessment methods, in a subsample.

\section{Methods}

\section{Study design and participants}

We used data from the Singapore PREconception Study of long-Term maternal and child Outcomes (S-PRESTO) study, a prospective cohort study, which aims to examine the role of exposures before conception and during pregnancy on maternal and offspring metabolic health outcomes in later life ${ }^{(20)}$. In the S-PRESTO study, non-pregnant women actively trying to conceive were enrolled between February 2015 and October 2017, from the community (through social media and radio advertisements, door to door leaflets, posters in public places and general practitioners clinics) and at the largest local public maternity unit (KK Women's and Children's Hospital, Singapore). They were of Chinese, Malay, Indian ethnicity or any combination of these three ethnicities, aged 18-45 years, planned to conceive within 1 year from recruitment and to reside in Singapore for the next 5 years. Women were excluded if (1) diagnosed with type I or type II diabetes, (2) on systemic steroids, anticonvulsants or treatment for HIV, hepatitis B or C in the past month prior to enrolment, (3) already pregnant at the first screening visit, (4) had already been trying to conceive for over 18 months and (5) were on assisted fertility treatment (except clomiphene and letrozole) or on hormonal contraception treatment in the past month prior to enrolment. Further details of the current study have been published elsewhere ${ }^{(20)}$.

\section{Socio-demographic, lifestyle and otber measures}

At enrolment, trained research staff conducted in-person interviews with participants and collected information including socio-demographic (e.g., age, highest educational attainment) and lifestyle behaviours (e.g., physical activity for the past $7 \mathrm{~d}$ and daily total sitting time (at work and during leisure), alcohol consumption) ${ }^{(21)}$. Taken in triplicates, mean values for anthropometry measures were recorded. These measures included weight (measured to the nearest $0 \cdot 1 \mathrm{~kg}$ using a SECA 803 weighing machine) and height (measured to the nearest $0.1 \mathrm{~cm}$ using a SECA 213 Portable Stadiometer), for the calculation of $\mathrm{BMI}^{(22)}$. BMI (expressed in $\mathrm{kg} / \mathrm{m}^{2}$ ) was calculated by dividing weight (in $\mathrm{kg}$ ) by squared height (in $\mathrm{m}^{2}$ ).

\section{Development of a maternal $F F Q$ for the Singapore PREconception Study of long-Term maternal and child Outcomes study}

A FFQ was developed based on locally validated FFQ previously used in nationally representative samples of adults $^{(23,24)}$. Additionally, food items commonly consumed during pregnancy and at postpartum were included. This information was obtained from a previous motheroffspring cohort (the Growing Up in Singapore Towards healthy Outcomes study - GUSTO), where food diaries 
and 24-h recalls from mothers during pregnancy and at postpartum were collected ${ }^{(25)}$. The semi-quantitative, interviewer-administered FFQ consisted of food and beverage items which captured details of cooking methods (such as boiled and deep fried) for certain food groups (e.g., vegetables, poultry, red meat and fish). Participants were instructed to consider their dietary intakes for the past month (prior to enrolment) when responding. For each food and beverage item, participants were asked to quantify their frequency of consumption in an open-ended format (using one of the four options: never/rarely, frequency per month, frequency per week or frequency per day) $)^{(26)}$ before specifying the average amount consumed at each instance. Picture aids of various food portion sizes (for items such as vegetables, poultry) and standard-sized household tableware were used during the interview. Frequencies of the various food and beverage items were standardised to daily intakes and multiplied by standard portions of food/beverage (in $\mathrm{g}$ ).

To estimate daily energy and nutrient intakes, a FFQ nutrient database was used. This database was generated using information from local food composition databases as well as information from the GUSTO maternal dietary records. For example, to obtain nutrient data for a FFQ item, a weighted average (weighting based on consumption frequency) was calculated based on the top three most commonly consumed foods under that item, from the GUSTO dietary records.

Upon completion of the FFQ during the enrolment visit, participants were given a 3DFD and guided on the recording of dietary intakes on two weekdays and one weekend day at home. To facilitate quantification of dietary intakes, picture aids of various food portion sizes and standard household tableware were included. Completed diaries were collected from participants at the subsequent study visit (second preconception visit occurring about 2-3 weeks later) and entered into a nutrient analysis software, Dietplan 7 (Forestfield Software Ltd), which contains local food composition databases.

\section{Food grouping (FFQ and 3-d food diary)}

The FFQ items were aggregated into forty-four pre-defined food groups (online supplementary material, Supplemental Table 1). To distinguish among the differing types of local staple consumed, distinct food groups for 'rice' (e.g., 'White rice', 'Flavoured rice' and 'Brown rice') and for 'noodles' (e.g., 'Noodles in soup' and 'Flavoured noodles') were created. Methods of cooking were also considered by differentiating foods cooked using healthier methods (lower fat content) $(\mathrm{H})$ such as boiled, steamed from those cooked using less healthy (higher fat content) (LH) methods such as deep fried, in coconut curry, and accordingly noted (e.g., poultry and meat $(\mathrm{H}) v$. poultry and meat (LH)). Food items recorded in the 3DFD were also classified into the identical forty-four food groups. For composite dishes, individual food items were identified and assigned to their respective food groups ${ }^{(27)}$. For all analyses involving the 3DFD, the averaged consumption of each food group was calculated across the $3 \mathrm{~d}$.

\section{Statistical analyses}

For the current study, 1007 women with completed FFQ and a subsample of 289 participants (29\% of 1007 women), who also completed 3DFD, were selected for analyses. To examine if the subsample ( $n$ 289) was representative of the larger sample ( $n$ 1007), the socio-demographic and lifestyle characteristics between the two groups were compared using Pearson $\chi^{2}$ tests.

Dietary patterns were first identified using EFA on the FFQ of the 1007 women with completed FFQ (Fig. 1, Step 1). Next, the reproducibility of these patterns was assessed using confirmatory factor analysis (CFA) in the FFQ and 3DFD of the subsample (Fig. 1, Step 2). To explain the suboptimal fit indices of the CFA models, exploratory factor analyses were next conducted and identified patterns from FFQ and 3DFD of the subsample were compared (Fig. 1, Steps 3 and 4). Further details are provided in the subsequent paragraphs.

\section{Identification of dietary patterns from the FFQ ( $n$ 1007)}

Suitability of the data for factor analysis was first assessed by the Kaiser-Meyer-Olkin measure of sampling adequacy and the Bartlett's test of sphericity ${ }^{(28,29)}$. EFA was used to identify a posteriori dietary patterns based on the forty-four food groups from women with completed FFQ ( $n$ 1007). To ensure that the dietary patterns derived were independent of one another and to improve interpretability ${ }^{(30)}$, varimax rotation was next performed. Based on the break point of the Scree plot, eigenvalue of more than one and factor interpretability ${ }^{(30)}$, an optimal number of factors was retained. Factor loadings, estimated using the principal factor method, represent correlation coefficients between food groups and their related dietary patterns; hence, a higher factor loading of a particular food group indicates greater contribution to a particular pattern. Subsequently, dietary pattern scores were calculated by summing the standardised daily intake of food groups weighted by the respective regressed factor loadings. Each participant had a score for each dietary pattern derived. Greater adherence to a particular pattern was indicated by a higher dietary pattern score.

Coefficients of congruence were determined to examine whether the patterns are congruent across sociodemographic factors ${ }^{(18,31)}$. These coefficients quantify the level of similarity between two dietary patterns compared, with values of above 0.50 considered as acceptable and values of close to 1 considered as excellent ${ }^{(3,18)}$. 


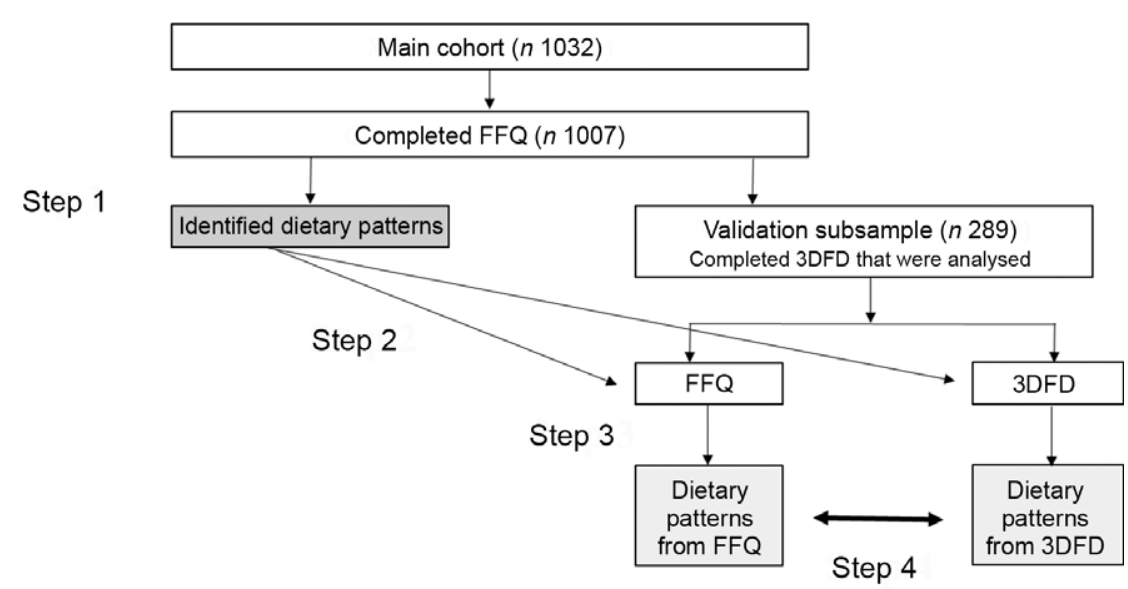

Fig. 1 Schematic of the analyses (Steps 1-4). Step 1: EFA was used for the generation of the dietary patterns from women with completed FFQ ( $n$ 1007). Step 2: The reproducibility of the identified patterns was assessed using CFA in the subsample ( $n$ 289). Step 3: EFA was used for the generation of dietary patterns in the subsample. This was conducted due to suboptimal global fit indices from the CFA analyses (especially the 3DFD). Step 4: Dietary patterns identified from each dietary assessment method are compared using congruence coefficients, quantifying the magnitudes of factor similarity between two patterns compared. EFA, exploratory factor analysis; CFA, confirmatory factor analysis

\section{Reproducibility of identified dietary patterns in the subsample using two dietary assessment methods ( $n$ 289)}

To assess the reproducibility of the identified patterns from the FFQ, CFA was applied on the FFQ and 3DFD of the subsample ${ }^{(15,19,32,33)}$. To retain the significant core of each identified pattern and ensure convergence is achieved, only food groups with factor loadings of at least 0.40 from the EFA from Step 1 were included ${ }^{(19,33)}$. Commonly reported fit indices of the CFA models (root mean square error of approximation (RMSEA), standardised root mean square residual, goodness of fit index, comparative fit index (CFI), Bayesian information criterion) were evaluated $^{(34)}$. The recommended cut-offs, indicating a good model fit, are values of RMSEA $<0.08$, standardised root mean square residual $<0 \cdot 08$, goodness of fit index $\geq 0.95$, CFI $\geq 0.90$ and smaller Bayesian information criterion values ${ }^{(34)}$.

\section{Dietary patterns in the subsample (n 289)}

EFA was subsequently conducted to identify dietary patterns derived from each dietary assessment method (FFQ and 3DFD) in the subsample. Coefficients of congruence were also determined to compare the identified patterns from each dietary assessment method. Additionally, Spearman correlation coefficients of the forty-four food groups (used for EFA) were calculated to compare intakes between the two dietary assessment methods.

Statistical analyses were conducted using STATA 14.0 (StataCorp LP) and the 'lavaan' package in $\mathrm{R}$ for CFA. A two-sided $P$-value of $<0.05$ indicates statistical significance.

\section{Results}

\section{Characteristics of participants}

Among the 1007 participants with available FFQ, a large proportion was below 35 years of age $(85.6 \%)$, of Chinese ethnicity $(71.7 \%)$, had higher educational attainment (62.4\%) and nulliparous (64.8\%) (online supplementary material, Supplemental Table 2). Participants who have never consumed alcohol or have never smoked made up $31.3 \%$ and $89.4 \%$ of the sample, respectively. While the majority had BMI within the normal range of $18.5-24.9 \mathrm{~kg} / \mathrm{m}^{2}(62.2 \%)$, a large proportion of participants led sedentary lifestyles as reflected by the low overall physical activity levels (66.5\% were inactive or minimally active) and long sitting hours ( $40 \cdot 1 \%$ sat for $>11$ h daily). The subsample ( $n$ 289) is representative of the cohort with FFQ data ( $n$ 1007) with largely similar characteristics, except the subsample having a larger proportion of older women $(P<0.001)$ and a larger proportion who were physically inactive $(P=0.003)$ (online supplementary material, Supplemental Table 2).

\section{Dietary patterns identified from FFQ (n 1007)}

The Kaiser-Meyer-Olkin measure of sampling adequacy was 0.73 for FFQ. The Bartlett's test of sphericity indicated that there was more than one underlying factor in the FFQ $(P<0.001)$. Both tests showed that the study sample was suitable for factor analysis. Three dietary patterns were identified from the FFQ: 'Fish, Poultry/Meat and Noodles', 'Fast Food and Sweetened Beverages' and 'Bread, Legumes and Dairy' (Table 1). These dietary patterns collectively explained $74 \%$ of the common variation in dietary intakes. The 'Fish, Poultry/Meat and Noodles' pattern was characterised by higher intakes of soups, fish, poultry/meat and noodles. The 'Fast Food and Sweetened Beverages' pattern was characterised by higher intakes of 


\section{Public Health Nutrition}

Table 1 Factor loadings for the dietary patterns identified from FFQ using exploratory factor analysis for women with completed FFQ ( $n$ 1007) and the subsample $(n$ 289)

\begin{tabular}{|c|c|c|c|c|c|c|c|c|}
\hline \multirow[b]{2}{*}{ Food groups } & \multicolumn{2}{|c|}{$\begin{array}{c}\text { Fish, Poultry/Meat and } \\
\text { Noodles }\end{array}$} & \multirow{2}{*}{$\frac{\text { Vegetables, Fruits, Poultry/Meat and Soups } \dagger}{3 \text { DFD (n 289) }}$} & \multicolumn{2}{|c|}{$\begin{array}{c}\text { Fast Food and Sweetened } \\
\text { Beverages* }\end{array}$} & \multicolumn{2}{|c|}{ Bread, Legumes and Dairy* } & \multirow{2}{*}{$\frac{\text { Bread, Pasta and Dairy }}{\text { 3DFD }(n \text { 289) }}$} \\
\hline & FFQ ( $n$ 1007) & $\mathrm{FFQ}(n 289)$ & & FFQ $(n$ 1007) & FFQ ( $n$ 289) & FFQ $(n 1007)$ & $\mathrm{FFQ}(n 289)$ & \\
\hline Soups & 0.50 & 0.54 & 0.39 & & & & & \\
\hline Dim sum & 0.42 & 0.41 & & & & & & \\
\hline Desserts in soup & 0.39 & 0.41 & & & & & & \\
\hline Oily and non-oily fish $(\mathrm{H})$ & 0.37 & 0.34 & 0.26 & & & & & \\
\hline Poultry and meat $(\mathrm{H})$ & 0.35 & 0.32 & 0.32 & & & & & \\
\hline Seafood and canned fish & 0.35 & 0.38 & & & & & & \\
\hline Eggs & 0.32 & & & & & & & \\
\hline Noodles in soup & 0.31 & & & & & & & \\
\hline Sausages/ham/bacon & & 0.39 & & 0.31 & & & & \\
\hline Pasta & & 0.31 & & & & & & 0.31 \\
\hline Innards & & 0.30 & & & & & & \\
\hline Fast food & & & -0.32 & 0.57 & 0.44 & & & \\
\hline Poultry and meat (LH) & & & & 0.48 & 0.43 & & & \\
\hline Sweetened beverages & & & & 0.47 & 0.35 & & & \\
\hline Flavoured rice & & & -0.25 & 0.44 & 0.37 & & & \\
\hline Fried snacks & & & & 0.38 & 0.37 & & & \\
\hline Flavoured noodles & & & -0.24 & 0.34 & & & & \\
\hline White rice & & & & 0.31 & & & & \\
\hline Fresh fruits & & & 0.40 & -0.30 & -0.34 & & & \\
\hline Vegetables $(\mathrm{H})$ & & & 0.58 & -0.37 & -0.53 & & & \\
\hline Oily and non-oily fish (LH) & & & & & 0.40 & & & \\
\hline Malted beverages & & & & & 0.35 & & & -0.22 \\
\hline Nuts and legumes & & & 0.32 & & & 0.45 & 0.52 & \\
\hline $\begin{array}{l}\text { Buns with sweet/savoury filling } \\
\text { bread (fried and steamed) }\end{array}$ & gs and ethnic & & & & & 0.44 & 0.37 & \\
\hline Vegetables (LH) & & & & & & 0.44 & 0.50 & \\
\hline $\begin{array}{l}\text { Savoury/sweet snacks (steam } \\
\text { pastries and cakes }\end{array}$ & ed/baked), & & & & & 0.43 & 0.42 & \\
\hline Chocolate & & & & & & 0.36 & & \\
\hline Bread spreads & & & & & & 0.34 & & 0.42 \\
\hline Full fat milk & & & & & & 0.34 & 0.42 & \\
\hline Yogurt/cultured/dairy drinks & & & & & & 0.33 & & 0.21 \\
\hline Plain/cream biscuits & & & & & & 0.31 & & \\
\hline Canned/dried fruits & & & 0.30 & & & 0.31 & 0.45 & \\
\hline Ice cream & & & & & & 0.30 & & 0.24 \\
\hline $\begin{array}{l}\text { Cereals, oats, cereal drinks an } \\
\text { cereals }\end{array}$ & nd other & & & & & & 0.38 & \\
\hline Vegetable salad & & & & & & & 0.33 & \\
\hline Soy products & & & 0.38 & & & & & \\
\hline White bread & & & & & & & & 0.62 \\
\hline Cheese & & & 0.21 & & & & & 0.35 \\
\hline Common variance explained & 23 & 17 & 19 & 25 & 17 & 26 & 17 & 13 \\
\hline
\end{tabular}

*Only factor loadings with absolute values of 0.30 and above were shown.

tOnly factor loadings with absolute values of 0.20 and above were shown. 
Table 2 Measures of global fit for confirmatory factor analysis of the identified patterns in the validation subsample $(n$ 289)

\begin{tabular}{lccc}
\hline Fit indices & Recommended cut-offs indicating a good fit & FFQ $(n$ 289) & 3DFD $(n$ 289) \\
\hline Root mean square error of approximation (RMSEA) & $<0.08$ & 0.070 & 0.065 \\
Standardised root mean square residual & $<0.08$ & 0.081 & 0.073 \\
Goodness of fit index & $\geq 0.95$ & 0.941 & 0.946 \\
Comparative fit index (CFI) & $>0.90$ & 0.787 & 0.343 \\
Bayesian information criterion & Smaller values indicate a better model fit & 27546 & 30923 \\
\hline
\end{tabular}

Table 3 Factor congruence between dietary patterns identified from the FFQ and 3DFD of the subsample $(n$ 289)

\begin{tabular}{llr}
\hline & Dietary patterns $(n$ 289) & Congruence coefficient \\
\hline FFQ & 3DFD & $0 \cdot 24$ \\
Fish, Poultry/Meat and Noodles & Vegetables, Fruits, Poultry/Meat and Soups & $0 \cdot 16$ \\
Fast Food and Sweetened Beverages & Bread, Pasta and Dairy & $-0 \cdot 64$ \\
& Vegetables, Fruits, Poultry/Meat and Soups & $0 \cdot 19$ \\
Bread, Legumes and Dairy & Bread, Pasta and Dairy & 0.30 \\
& Vegetables, Fruits, Poultry/Meat and Soups & 0.31 \\
\hline
\end{tabular}

fast food, sweetened beverages and processed meat, and lower intakes of cooked vegetables and fresh fruits. The 'Bread, Legumes and Dairy' pattern consisted of mainly plant-based foods and dairy products such as higher intakes of nuts, legumes, full fat milk, yogurt and dairybased drinks. These patterns were reasonably congruent across socio-demographic factors such as maternal age and ethnicity among the 1007 women (online supplementary material, Supplemental Table 3).

\section{Reproducibility of identified dietary patterns in the subsample using two dietary assessment metbods ( $n$ 289)}

On the basis of several fit indices (especially the CFI and Bayesian information criterion), the identified patterns ('Fish, Poultry/Meat and Noodles', 'Fast Food and Sweetened Beverages' and 'Bread, Legumes and Dairy') had a better model fit in the FFQ as compared with the 3DFD of the subsample (CFI for $F F Q=0.79$; CFI for $3 \mathrm{DFD}=0.34$ and smaller Bayesian information criterion value for the FFQ compared with the 3DFD) (Table 2). To explain the poorer reproducibility of the identified patterns in the 3DFD, dietary patterns were identified for each dietary assessment method in the subsample.

\section{Dietary patterns in the subsample (n 289)}

Dietary patterns ('Fish, Poultry/Meat and Noodles', 'Fast Food and Sweetened Beverages' and 'Bread, Legumes and Dairy') similar to those in the FFQ of the large sample were identified in the FFQ of the subsample. They explained $51 \%$ of the common variance in dietary intakes. Conversely, two patterns, explaining $32 \%$ of the common variance in dietary intakes, were observed from the 3DFD of the subsample. They appeared to be variants of two dietary patterns found in the FFQ and have been named as 'Vegetables, Fruits, Poultry/Meat and Soups' and 'Bread, Pasta and Dairy' (Table 1). In this subsample, a third 3DFD pattern was omitted due to poor interpretability. The third FFQ 'Fast Food and Sweetened Beverages' pattern did not resemble any of those identified from the 3DFD.

The 'Vegetables, Fruits, Poultry/Meat and Soups' pattern of the 3DFD appeared somewhat similar to the FFQ 'Fish, Poultry/Meat and Noodles' pattern except that it included additional key food groups such as vegetables, fruits and soya products. Similarly, the 'Bread, Pasta and Dairy' of the 3DFD shared similarities with the FFQ 'Bread, Legumes and Dairy' pattern in the subsample, except that pasta, white bread and cheese were included as additional food groups. However, the congruence coefficients of patterns identified in the subsample were below the acceptable cut-off of 0.50 (ranged from -0.64 (FFQ 'Fast Food and Sweetened Beverages' and 3DFD 'Vegetables, Fruits, Poultry/Meat and Soups') to 0.31 (FFQ 'Bread, Legumes and Dairy' and 3DFD 'Bread, Pasta and Dairy')] (Table 3), suggesting that patterns identified from the FFQ and 3DFD of the subsample cannot be considered similar.

In the subsample, a comparison of the food group intakes from the two dietary assessment methods explained the dissimilar patterns identified from the FFQ and 3DFD (Table 4). In general, the FFQ captured a wider variety of foods consumed as compared with the 3DFD, with a larger number of food groups having median intakes of null in the latter. Spearman correlation coefficient was lowest for desserts in soup (0.12) and highest for coffee/ tea/decaffeinated drinks (0.66).

\section{Discussion}

Three dietary patterns ('Fish, Poultry/Meat and Noodles', 'Fast Food and Sweetened Beverages' and 'Bread, 
Table 4 Comparison of intakes of food groups for women with completed FFQ $(n$ 1007) and the subsample $(n$ 289)

\begin{tabular}{|c|c|c|c|c|c|c|c|}
\hline \multirow[b]{2}{*}{ Food groups (g/d) } & \multicolumn{2}{|c|}{$\begin{array}{l}\text { Women with } \\
\text { available } \\
\text { FFQ }(n 1007) \\
\end{array}$} & \multicolumn{4}{|c|}{ Women in the subsample $(n$ 289) } & \multirow[b]{2}{*}{ Spearman rho† } \\
\hline & Median & IQR & FFQ median & FFQ IQR & 3DFD median & 3DFD IQR & \\
\hline \multicolumn{8}{|l|}{ Fish, Poultry/Meat and Noodles } \\
\hline Coffee/tea/decaffeinated drinks & 179 & 277 & 179 & 286 & 150 & 283 & 0.66 \\
\hline Brown rice & 0 & 29 & 0 & 29 & 0 & 0 & 0.49 \\
\hline Soya products & 27 & 42 & 22 & 40 & 0 & 28 & 0.38 \\
\hline Poultry and meat $(\mathrm{H})$ & 76 & 74 & 73 & 62 & 30 & 50 & 0.33 \\
\hline Eggs & 27 & 33 & 21 & 25 & 16 & 33 & 0.28 \\
\hline Oily and non-oily fish $(\mathrm{H})$ & 26 & 42 & 28 & 42 & 10 & 40 & 0.28 \\
\hline Porridge & 20 & 62 & 26 & 70 & 0 & 0 & 0.27 \\
\hline Soups & 38 & 80 & 33 & 67 & 47 & 118 & 0.26 \\
\hline Sausages/ham/bacon & 2 & 4 & 2 & 4 & 0 & 0 & 0.25 \\
\hline Noodles in soup & 120 & 181 & 106 & 179 & 29 & 193 & 0.24 \\
\hline Dim sum & 7 & 11 & 10 & 11 & 0 & 20 & 0.21 \\
\hline Pasta & 6 & 12 & 6 & 12 & 0 & 0 & 0.20 \\
\hline Seafood and canned fish & 5 & 8 & 5 & 6 & 0 & 10 & 0.17 \\
\hline Innards & 0 & 2 & 0 & 2 & 0 & 0 & 0.12 \\
\hline Desserts in soup & 18 & 34 & 20 & 40 & 0 & 0 & 0.12 \\
\hline \multicolumn{8}{|l|}{ Fast Food and Sweetened Beverages } \\
\hline Malted beverages & 27 & 71 & 27 & 71 & 0 & 83 & 0.43 \\
\hline Fresh fruits & 97 & 133 & 101 & 126 & 28 & 104 & 0.40 \\
\hline White bread & 9 & 24 & 9 & 25 & 0 & 20 & 0.39 \\
\hline Sweetened beverages & 36 & 98 & 36 & 98 & 83 & 183 & 0.37 \\
\hline White rice & 143 & 129 & 120 & 143 & 67 & 100 & 0.35 \\
\hline Vegetables $(\mathrm{H})$ & 166 & 161 & 158 & 154 & 62 & 74 & 0.34 \\
\hline Flavoured noodles & 53 & 96 & 50 & 85 & 83 & 173 & 0.27 \\
\hline Flavoured rice & 24 & 28 & 23 & 28 & 0 & 100 & 0.22 \\
\hline Fast foods & 36 & 44 & 35 & 40 & 0 & 33 & 0.22 \\
\hline Fried snacks & 5 & 9 & 5 & 9 & 0 & 11 & 0.18 \\
\hline Poultry and meat (LH) & 11 & 23 & 8 & 18 & 0 & 30 & 0.14 \\
\hline Oily and non-oily fish (LH) & 0 & 7 & 0 & 8 & 0 & 0 & $-0.01^{*}$ \\
\hline \multicolumn{8}{|l|}{ Bread, Legumes and Dairy } \\
\hline Cereals, oats, cereal drinks and other cereals & 9 & 36 & 9 & 34 & 0 & 18 & 0.43 \\
\hline Yogurt/cultured/dairy drinks & 28 & 64 & 29 & 64 & 0 & 33 & 0.41 \\
\hline Bread spreads & 2 & 4 & 2 & 4 & 0 & 3 & 0.40 \\
\hline Vegetable salad & 10 & 27 & 10 & 27 & 0 & 13 & 0.40 \\
\hline Cheese & 1 & 3 & 1 & 3 & 0 & 0 & 0.36 \\
\hline Wholemeal bread & 8 & 21 & 8 & 22 & 0 & 0 & 0.36 \\
\hline Plain/cream biscuits & 5 & 14 & 5 & 14 & 0 & 11 & 0.34 \\
\hline Full fat milk & 9 & 71 & 0 & 71 & 0 & 0 & 0.34 \\
\hline $\begin{array}{l}\text { Buns with sweet/savoury fillings and } \\
\text { ethnic bread (fried and steamed) }\end{array}$ & 21 & 35 & 19 & 29 & 11 & 39 & 0.34 \\
\hline Nuts and legumes & 1 & 5 & 1 & 5 & 0 & 2 & 0.30 \\
\hline Low fat milk/formula milk & 0 & 36 & 0 & 36 & 0 & 0 & 0.24 \\
\hline Fruit juice & 21 & 43 & 16 & 43 & 0 & 0 & 0.21 \\
\hline Chocolate & 1 & 2 & 1 & 1 & 0 & 0 & 0.19 \\
\hline Vegetables (LH) & 0 & 10 & 0 & 8 & 0 & 0 & 0.16 \\
\hline Canned/dried fruits & 0 & 1 & 0 & 1 & 0 & 0 & 0.14 \\
\hline Ice cream & 5 & 8 & 5 & 11 & 0 & 0 & $0.11^{*}$ \\
\hline $\begin{array}{l}\text { Savoury/sweet snacks (steamed/baked), } \\
\text { pastries and cakes }\end{array}$ & 11 & 16 & 11 & 16 & 9 & 38 & $0 \cdot 11^{*}$ \\
\hline
\end{tabular}

$I Q R$, interquartile range; $\mathrm{H}$, healthy, $\mathrm{LH}$, less healthy.

${ }^{*} P>0.05$.

†Spearman correlation coefficients were calculated between intakes from the FFQ and 3DFD in the subsample ( $n$ 289)

Legumes and Dairy') were identified from the FFQ. In the subsample, these patterns were reproducible in the FFQ, but less so in the 3DFD, where two variant patterns emerged. In this cohort, a posteriori dietary patterns identified from the FFQ were reproducible and reasonably congruent across several socio-demographic factors within this cohort of women planning pregnancy.

\section{Dietary patterns identified from FFQ (n 1007)}

The 'Fish, Poultry/Meat and Noodles' pattern in the S-PRESTO cohort is in line with our local diets consisting of higher carbohydrates and protein, with low intakes of fruits and vegetables ${ }^{(35,36)}$. This contrasts to the patterns reported among Spanish or Australian women planning pregnancy where vegetables or fruits tended to be eaten in combination with 
animal protein (e.g., 'Vegetables and Meat' pattern (consisting of a variety of vegetables, meat) ${ }^{(3)}$, 'High-protein/ fruit' (consisting of fish, meat, fresh fruits) and 'Fruit and Low-fat Dairy' (consisting of fresh fruits, yogurt) ${ }^{(16,17)}$ ).

The 'Fast Food and Sweetened Beverages' pattern in the S-PRESTO cohort consisted of discretionary foods which are high in fat and sugar (e.g., fast food, sweetened beverages, fried snacks). This is similar to the 'Sweetened Beverages and Sugars' (consisting of sweetened beverages, sugars, low intakes of vegetables, fresh fruits), 'Meat, High-fat and Sugar' (consisting of cakes, sweet biscuits, meat pies) and 'High-fat/sugar/takeaway' (consisting of takeaway foods, potato chips, refined grains) patterns observed among Australian and Spanish women planning pregnancy ${ }^{(3,16,17)}$.

A unique 'Bread, Legumes and Dairy' pattern, made up of foods mainly from plant sources (e.g., nuts and legumes, buns and ethnic bread and a variety of cooked vegetables prepared using coconut curry), was observed in the S-PRESTO cohort. To our knowledge, this combination of foods has not been reported in other preconception cohorts to date, probably due to the lack of sampling on minority ethnic groups (e.g., Malay and Indian ethnicities). Taken together, while the dietary patterns of S-PRESTO women shared common foods that were identified in other populations of women planning pregnancy, they highlighted diverse consumption patterns within an Asian setting.

\section{Reproducibility of identified dietary patterns in the subsample using two dietary assessment methods ( $n$ 289)}

CFA has been used in nutrition studies to examine the robustness of empirically derived patterns ${ }^{(15,19,32,37)}$, and by reducing the subjectivity and ambiguity involved in exploratory approaches ${ }^{(19,33,37)}$. In line with these studies with reported CFI values of 0.64-0.71 and RMSEA values of $0 \cdot 105-0 \cdot 805^{(15,18)}$, we found that the identified patterns from the large sample were reproducible in the subsample when the same dietary assessment method (FFQ) was used $(\mathrm{CFI}=0.787$, RMSEA $=0.070)$. Unlike two other studies, where $7-d$ weighed diet record readily identified patterns obtained from the $\mathrm{FFQ}^{(15)}$, the identified patterns were less reproducible when $3 \mathrm{DFD}$ was used in the current study $(\mathrm{CFI}=0.343$, RMSEA $=0.065)$. A shorter, limited reference period ( $3 \mathrm{~d}$ ) and a less precise measurement of intakes (non-weighed) in the current study could have contributed to the poorer reproducibility of the identified patterns in the 3DFD of the subsample. An exploration of the dietary patterns captured by each dietary assessment method in the subsample further substantiated these findings.

\section{Dietary patterns in the subsample (n 289)}

Differences in the patterns identified could be largely due to the fundamental differences between the two dietary assessment methods. Firstly, the FFQ is a closed-ended checklist of foods that captured estimated intakes of each given item, while the 3DFD is a detailed, open-ended record of the actual foods consumed. During the 3DFD dietary analyses, recorded foods and individual components of composite foods are often re-coded to match the closest corresponding FFQ food items. This lack of 'one-to-one correspondence' between foods captured by the FFQ and those in the 3DFD could have led to the differing patterns observed ${ }^{(38)}$.

Secondly, the dietary assessment methods had differing reference periods for the recording of intakes (FFQ: intakes in the past month; 3DFD: $3 \mathrm{~d}$ of a selected week after FFQ administration), which is likely to have resulted in a larger variation of foods captured by the FFQ in the subsample (FFQ: $51 \%$; 3DFD: $32 \%$ of the variation explained). As changes in diet were unlikely within the short interval between the administration of the FFQ and 3DFD, differences in intakes captured could be largely due to the dietary assessment methods used ${ }^{(38)}$.

Thirdly, women planning pregnancy could have reduced their intakes of discretionary foods (such as fast food, fried snacks) upon intending to conceive ${ }^{(39)}$. These foods (mainly found in the FFQ 'Fast Food and Sweetened Beverages' pattern) are thus less likely to be consumed over the course of $3 \mathrm{~d}$, hence were not captured by the 3DFD. In view of the differences between the two dietary assessment methods, we suggest the use of the FFQ-derived dietary patterns (instead of 3DFD ones), based on their better reproducibility and ability to reflect habitual intakes, for future diet-related investigations on health outcomes in this cohort.

Strengths of the current study include having a relatively large sample size ( $n$ 289) as compared with other dietary validation studies with sample sizes ranging from 92 to 203 participants $^{(9,13,14,40-42)}$ and the reproducibility of these patterns was examined using CFA, giving additional credence to the results ${ }^{(43)}$. However, several limitations are worth noting. Firstly, we regarded the FFQ patterns to be reflective of the habitual dietary intakes in this cohort, though it is also prone to reporting errors and biases. Nonetheless, the FFQ has been widely used as a measure for diet association studies ${ }^{(38)}$. Secondly, it is possible that the behavioural effect of reporting dietary intakes may obscure the true dietary intakes of participants and hence the patterns identified (e.g., socially desirable reporting of diets during interviewer-administered FFQ or simplifying food intakes to reduce reporting burden for the 3DFD) ${ }^{(44)}$. While this cannot be avoided entirely, we expect both dietary assessment methods to be similarly affected. Thirdly, no or low intakes for specific food groups could be a concern when identifying the patterns, especially so for the 3DFD. To address this, we performed EFA on the thirteen food groups where at least $50 \%$ of the women had intakes recorded in the $3 \mathrm{DFD}$. However, only two patterns were identified and they provided little information on the overall diets. As such, the number of food groups 
used in all analyses was standardised for consistency, an approach that was adopted by similar studies ${ }^{(8)}$.

\section{Conclusion}

We have identified three dietary patterns ('Fish, Poultry/ Meat and Noodles', 'Fast Food and Sweetened Beverages' and 'Bread, Legumes and Dairy') among Asian women planning pregnancy. In general, the three dietary patterns assessed by the FFQ were reproducible and reasonably congruent across several sociodemographic characteristics, providing a basis for future investigations on the overall diet and health outcomes in this cohort.

\section{Acknowledgements}

Acknowledgements: We would like to extend our gratitude to the S-PRESTO participants and the S-PRESTO study group, which includes Anne Eng Neo Goh, Anne RifkinGraboi, Anqi Qiu, Bee Wah Lee, Bernard Chern, Bobby Cheon, Christiani Jeyakumar Henry, Ciaran Gerard Forde, Claudia Chi, Doris Fok, Elaine Quah, Elizabeth Tham, Evelyn Chung Ning Law, Evelyn Xiu Ling Loo, Fabian Yap, Faidon Magkos, Falk Mueller-Riemenschneider, George Seow Heong Yeo, Helen Yu Chen, Heng Hao Tan, Hugo PS van Bever, Izzuddin Bin Mohd Aris, Jerry Kok Yen Chan, Joanne Yoong, Joao N Ferreira., Jonathan Tze Liang Choo, Jonathan Y Bernard, Keith M Godfrey, Kenneth Kwek, Kok Hian Tan, Kuan Jin Lee, Lieng Hsi Ling, Ling Wei Chen, Lourdes Mary Daniel, Lynette Pei-Chi Shek, Marielle V. Fortier, Mary Foong-Fong Chong, Mei Chien Chua, Melvin Leow, Michael Meaney, Mya Thway Tint, Neerja Karnani, Ngee Lek, Oon Hoe Teoh, Peter D Gluckman, Queenie Ling Jun Li, See Ling Loy, Sendhil Velan, Seng Bin Ang, Sharon Ng, Shephali Tagore, ShiaoYng Chan, Shirong Cai, Shu E Soh, Sok Bee Lim, Stella Tsotsi, Stephen Chin-Ying Hsu, Sue Anne Toh, Teng Hong Tan, Tong Wei Yew, Victor Samuel Rajadurai, Wee Meng Han, Wei Wei Pang, Yap Seng Chong, Yin Bun Cheung, Yiong Huak Chan, Yung Seng Lee. Financial support: This research is supported by the Singapore National Research Foundation (NRF) under its Translational and Clinical Research (TCR) Flagship Programme and administered by the Singapore Ministry of Health's National Medical Research Council (NMRC), Singapore-NMRC/TCR/ 004-NUS/2008; NMRC/TCR/012-NUHS/2014. Additional funding is provided by the Singapore Institute for Clinical Sciences, Agency for Science, Technology and Research (A*STAR), Singapore. K.M.G. is supported by the National Institute for Health Research through the NIHR Southampton Biomedical Research Centre and the European Union's Seventh Framework Programme (FP7/2007-2013), project EarlyNutrition ( $n^{\circ} 289346$ ). J.K.Y.C. is supported by the NMRC Clinician Scientist
Award (NMRC/CSA(SI)/008/2016). Conflict of interest: Y.S.C., K.M.G. and S.Y.C. are part of an academic consortium that has received research funding from Abbott, Nutrition, Nestle and Danone. No conflict of interest was declared by the other authors. Authorship: Y.S.C., K.M.G., L.P.C.S., S.Y.C. and J.K.Y.C. designed and led the S-PRESTO cohort study. M.T.C. and M.N.M.A. contributed to the FFQ and 3DFD dietary data collection, cleaning and analysis. Hiromi Koh provided statistical advice for this manuscript. S.X.L. contributed to the FFQ data cleaning, analysed the data and wrote the paper with intellectual inputs from M.F.F.C. All authors read and approved the manuscript. Ethics of human subject participation: The S-PRESTO study is conducted according to the guidelines as stated in the Declaration of Helsinki and all procedures involving research study participants were approved by the local SingHealth Centralized Institutional Review Board (reference 2019/2143). The study is registered on the ClinicalTrials.gov database provided by the US National Library of Medicine under the identifier NCT03531658. Written consent was obtained from all participants.

\section{Supplementary material}

For supplementary material accompanying this paper visit https://doi.org/10.1017/S1368980021001178

\section{References}

1. Chen L-W, Low YL, Fok D et al. (2014) Dietary changes during pregnancy and the postpartum period in Singaporean Chinese, Malay and Indian women: the GUSTO birth cohort study. Public Health Nutr 17, 1930-1938.

2. Hillier SE \& Olander EK (2017) Women's dietary changes before and during pregnancy: a systematic review. Midwifery 49, 19-31.

3. Cuco G, Fernandez-Ballart J, Sala J et al. (2006) Dietary patterns and associated lifestyles in preconception, pregnancy and postpartum. Eur J Clin Nutr 60, 364-371.

4. Olson CM (2005) Tracking of food choices across the transition to motherhood. J Nutr Educ Behav 37, 129-136.

5. Tovar A, Kaar JL, McCurdy K et al. (2019) Maternal vegetable intake during and after pregnancy. BMC Pregnancy Childbirth 19, 267.

6. Hu FB (2002) Dietary pattern analysis: a new direction in nutritional epidemiology. Curr Opin Lipidol 13, 3-9.

7. Reedy J, Subar AF, George SM et al. (2018) Extending methods in dietary patterns research. Nutrients 10, 571.

8. Edefonti V, De Vito R, Dalmartello M et al. (2020) Reproducibility and validity of a posteriori dietary patterns: a systematic review. Adv Nutr 11, 293-326.

9. Asghari G, Rezazadeh A, Hosseini-Esfahani F et al. (2012) Reliability, comparative validity and stability of dietary patterns derived from an FFQ in the Tehran Lipid and Glucose Study. BrJ Nutr 108, 1109-1117.

10. Crozier SR, Inskip HM, Godfrey KM et al. (2008) Dietary patterns in pregnant women: a comparison of food-frequency questionnaires and $4 \mathrm{~d}$ prospective diaries. Br J Nutr 99 , 869-875. 
11. Hu FB, Rimm E, Smith-Warner SA et al. (1999) Reproducibility and validity of dietary patterns assessed with a food-frequency questionnaire. Am J Clin Nutr 69, 243-249.

12. Khani BR, Ye W, Terry P et al. (2004) Reproducibility and validity of major dietary patterns among Swedish women assessed with a food-frequency questionnaire. J Nutr 134, $1541-1545$

13. Loy SL \& Jan Mohamed HJ (2013) Relative validity of dietary patterns during pregnancy assessed with a food frequency questionnaire. Int J Food Sci Nutr 64, 668-673.

14. Okubo H, Murakami K, Sasaki S et al. (2010) Relative validity of dietary patterns derived from a self-administered diet history questionnaire using factor analysis among Japanese adults. Public Health Nutr 13, 1080-1089.

15. Togo P, Heitmann BL, Sorensen TI et al. (2003) Consistency of food intake factors by different dietary assessment methods and population groups. Br J Nutr 90, 667-678.

16. Schoenaker DA, Soedamah-Muthu SS, Callaway LK et al. (2015) Prepregnancy dietary patterns and risk of developing hypertensive disorders of pregnancy: results from the Australian Longitudinal Study on Women's Health. Am J Clin Nutr 102, 94-101.

17. Grieger JA, Grzeskowiak LE \& Clifton VL (2014) Preconception dietary patterns in human pregnancies are associated with preterm delivery. J Nutr 144, 1075-1080.

18. Judd SE, Letter AJ, Shikany JM et al. (2015) Dietary patterns derived using exploratory and confirmatory factor analysis are stable and generalizable across race, region, and gender subgroups in the REGARDS study. Front Nutr 1, 29-29.

19. Lau C, Glümer C, Toft U et al. (2008) Identification and reproducibility of dietary patterns in a Danish cohort: the Inter99 study. Br J Nutr 99, 1089-1098.

20. Loo EXL, Soh SE, Loy SL et al. (2021) Cohort profile: Singapore preconception study of long-term maternal and child outcomes (S-PRESTO). Eur J Epidemiol 36, 129-142.

21. Bernard JY, Ng S, Natarajan P et al. (2019) Associations of physical activity levels and screen time with oral glucose tolerance test profiles in Singaporean women of reproductive age actively trying to conceive: the S-PRESTO study. Diabet Med 36, 888-897.

22. Loy SL, Cheung YB, Soh SE et al. (2018) Female adiposity and time-to-pregnancy: a multiethnic prospective cohort. Hum Reprod 33, 2141-2149.

23. Deurenberg-Yap M, Li T, Tan WL et al. (2000) Validation of a semiquantitative food frequency questionnaire for estimation of intakes of energy, fats and cholesterol among Singaporeans. Asia Pac J Clin Nutr 9, 282-288.

24. Neelakantan N, Whitton C, Seah S et al. (2016) Development of a semi-quantitative food frequency questionnaire to assess the dietary intake of a multi-ethnic urban Asian population. Nutrients 8, 528 .

25. Chia AR, de Seymour JV, Colega M et al. (2016) A vegetable, fruit, and white rice dietary pattern during pregnancy is associated with a lower risk of preterm birth and larger birth size in a multiethnic Asian cohort: the Growing Up in Singapore Towards healthy Outcomes (GUSTO) cohort study. Am J Clin Nutr 104, 1416-1423.

26. Block G, Hartman AM, Dresser CM et al. (1986) A data-based approach to diet questionnaire design and testing. $\mathrm{Am} \mathrm{J}$ Epidemiol 124, 453-469.
27. Ambrosini GL, O'Sullivan TA, de Klerk NH et al. (2011) Relative validity of adolescent dietary patterns: a comparison of a FFQ and 3 d food record. Br J Nutr 105, 625-633.

28. Alvin RC (2002) Factor analysis. In Methods of Multivariate Analysis, 2nd ed, pp. 408-450. New York: John Wiley and Sons, Inc.

29. Hair JF, Black WC \& Babin BJ (2010) Chapter 3 - Factor analysis. In Multivariate Data Analysis: A Global Perspective. London: Pearson Education.

30. Newby PK \& Tucker KL (2004) Empirically derived eating patterns using factor or cluster analysis: a review. Nutr Rev 62, 177-203.

31. Lorenzo-Seva U \& Ten Berge JM (2006) Tucker's congruence coefficient as a meaningful index of factor similarity. Methodology (Gott) 2, 57-64.

32. Newby PK, Weismayer C, Akesson A et al. (2006) Long-term stability of food patterns identified by use of factor analysis among Swedish women. J Nutr 136, 626-633.

33. Schulze MB, Hoffmann K, Kroke A et al. (2003) Risk of hypertension among women in the EPIC-Potsdam Study: comparison of relative risk estimates for exploratory and hypothesis-oriented dietary patterns. Am J Epidemiol 158, 365-373.

34. Hooper D, Coughlan J \& Mullen, M (2008) Structural equation modelling: guidelines for determining model fit. EJBRM 6, 53-59.

35. Health Promotion Board Singapore (2018) National Nutrition Survey 2018 Shows Gradual Improvements in Singaporeans' Dietary Habits; available at https://www.hpb.gov.sg/article/ national-nutrition-survey-2018-shows-gradual-improvementsin-singaporeans-dietary-habits (accessed January 2021).

36. Health Promotion Board Singapore (2011) Report of the National Nutrition Survey 2010; available at https://www. hpb.gov.sg/docs/default-source/pdf/nns-2010-report.pdf? sfvrsn=18e3f172_2 (accessed February 2021).

37. Maskarinec G, Novotny R \& Tasaki K (2000) Dietary patterns are associated with body mass index in multiethnic women. J Nutr 130, 3068-3072.

38. Willett W \& Hatch E (2013) Chapter 6: Reproducibility and validity of food frequency questionnaires. In Nutritional Epidemiology, 3rd ed. [electronic resource] Oxford: Oxford University Press

39. Wise LA, Wesselink AK, Hatch EE et al. (2020) Changes in behavior with increasing pregnancy attempt time: a prospective cohort study. Epidemiology 31, 659-667.

40. Beck KL, Kruger R, Conlon CA et al. (2012) The relative validity and reproducibility of an iron food frequency questionnaire for identifying iron-related dietary patterns in young women. J Acad Nutr 112, 1177-1187.

41. Hong X, Ye Q, Wang Z et al. (2016) Reproducibility and validity of dietary patterns identified using factor analysis among Chinese populations. Br J Nutr 116, 842-852.

42. Liu X, Wang X, Lin S et al. (2015) Reproducibility and validity of a food frequency questionnaire for assessing dietary consumption via the dietary pattern method in a Chinese rural population. PLoS One 10, e0134627.

43. Cade JE, Burley VJ, Warm DL et al. (2004) Food-frequency questionnaires: a review of their design, validation and utilisation. Nutr Res Rev 17, 5-22.

44. Livingstone MBE \& Black AE (2003) Markers of the validity of reported energy intake. J Nutr 133, 895S-920S. 